\title{
Kidney and cystic volume imaging for disease presentation and progression in the cat autosomal dominant polycystic kidney disease large animal model
}

Yoshihiko $\mathrm{Yu}^{1,2 \dagger}{ }^{1}$, Kate L. Shumway ${ }^{1 \dagger}$, Jodi S. Matheson ${ }^{1}$, Marie E. Edwards ${ }^{3}$, Timothy L. Kline ${ }^{3,4}$ and Leslie A. Lyons ${ }^{1 *}$ (D)

\begin{abstract}
Background: Approximately 30\% of Persian cats have a c.10063C > A variant in polycystin 1 (PKD1) homolog causing autosomal dominant polycystic kidney disease (ADPKD). The variant is lethal in utero when in the homozygous state and is the only ADPKD variant known in cats. Affected cats have a wide range of progression and disease severity. However, cats are an overlooked biomedical model and have not been used to test therapeutics and diets that may support human clinical trials. To reinvigorate the cat as a large animal model for ADPKD, the efficacy of imaging modalities was evaluated and estimates of kidney and fractional cystic volumes (FCV) determined.
\end{abstract}

Methods: Three imaging modalities, ultrasonography, computed tomography $(C T)$, and magnetic resonance imaging examined variation in disease presentation and disease progression in 11 felines with ADPKD. Imaging data was compared to well-known biomarkers for chronic kidney disease and glomerular filtration rate. Total kidney volume, total cystic volume, and FCV were determined for the first time in ADPKD cats. Two cats had follow-up examinations to evaluate progression.

Results: FCV measurements were feasible in cats. CT was a rapid and an efficient modality for evaluating therapeutic effects that cause alterations in kidney volume and/or FCV. Biomarkers, including glomerular filtration rate and creatinine, were not predictive for disease progression in feline ADPKD. The wide variation in cystic presentation suggested genetic modifiers likely influence disease progression in cats. All imaging modalities had comparable resolutions to those acquired for humans, and software used for kidney and cystic volume estimates in humans proved useful for cats.

Conclusions: Routine imaging protocols used in veterinary medicine are as robust and efficient for evaluating ADPKD in cats as those used in human medicine. Cats can be identified as fast and slow progressors, thus, could assist with genetic modifier discovery. Software to measure kidney and cystic volume in human ADPKD kidney studies is applicable and efficient in cats. The longer life and larger kidney size span than rodents, similar genetics, disease presentation and progression as humans suggest cats are an efficient biomedical model for evaluation of ADPKD therapeutics.

Keywords: ADPKD, ESRD, Feline, PKD1

\footnotetext{
* Correspondence: lyonsla@missouri.edu

${ }^{\dagger}$ Yoshihiko Yu and Kate L. Shumway contributed equally to this work.

'Department of Veterinary Medicine and Surgery, College of Veterinary

Medicine, University of Missouri, Columbia, MO, USA

Full list of author information is available at the end of the article
}

(c) The Author(s). 2019 Open Access This article is distributed under the terms of the Creative Commons Attribution 4.0 International License (http://creativecommons.org/licenses/by/4.0/), which permits unrestricted use, distribution, and reproduction in any medium, provided you give appropriate credit to the original author(s) and the source, provide a link to the Creative Commons license, and indicate if changes were made. The Creative Commons Public Domain Dedication waiver (http://creativecommons.org/publicdomain/zero/1.0/) applies to the data made available in this article, unless otherwise stated. 


\section{Background}

Autosomal dominant polycystic kidney disease (ADPKD) is one of the most commonly inherited disorders in humans, with an estimated prevalence of $1: 400$ to $1: 1$, 000 [1]. ADPKD is the fourth most common cause of renal replacement therapy (i.e., dialysis or transplant) [2-4] and is generally diagnosed by imaging of the kidney using ultrasonography (US), computed tomography (CT), or magnetic resonance imaging (MRI). For ADPKD, approximately $75-85 \%$ of cases are caused by variants in PKD1, which encodes the protein known as polycystin-1 [5-7]. Most human families with ADPKD have novel variants and over 1,273 causal variants for PKD1 are catalogued in the Autosomal Dominant Polycystic Kidney Disease Mutation Database: PKDB [8]. Because of this genetic heterogeneity, cohorts with the same PKD1 variant or cohorts with the same genetic backgrounds are limited in humans, which inhibits the power of studies focused on genetic modifiers that would influence interfamilial, intrafamilial and sex differences in disease progression and responses to therapeutics. Genetic screening is also complicated in humans since PKD1 includes 46 exons, has a large, $\sim 14 \mathrm{~kb}$ mRNA [9] spanning a $47.2 \mathrm{~kb}$ genomic region, and six pseudogenes are present in the human genome $[10,11]$.

ADPKD is widely recognized as the most commonly inherited renal disease in the domestic cat, specifically cats of the Persian breed [12-16]. The feline PKD1 variant (c.10063C > A) causes a stop codon at position 3284 in exon 29 (C3284X) [17] and is the only variant causing ADPKD in cats known to date. This variant is found in Persian-related breeds as well [17-19]. Hepatic and pancreatic cysts are present in some cats with ADPKD [14, $16,20]$, however, hypertension is noted to be minor [21], and other vascular or systemic complications are not documented. Although many ADPKD cats remain subclinical through-out their lives, some show rapid disease progression, developing chronic kidney disease (CKD) secondary to ADPKD, and succumb to disease within 7 years of life or earlier, which is only mid-life for a cat $[16,22]$. These younger cats that succumb to disease have consistent disease progressing to humans who have truncating $P K D 1$ variants [5]. Cats should be instrumental for identifying genetic modifiers of cystic progression and for deciphering variation in therapeutic responses since the solitary causal variant and control of the genetic background via colony of ADPKD cats will reduce variables in the analyses.

US, CT, and MRI are commonly used imaging modalities to evaluate disease progression and the therapeutic efficacy in humans with ADPKD [23], however, detailed and comparative imaging in feline ADPKD using different modalities is limited. US is routinely used to diagnose feline ADPKD, however, a study assessing the progression of the disease over time (approximately 1 year) showed an apparent improvement in a small number of cats [24], thus, the accuracy of US is questionable. In humans, US is used to screen ADPKD-suspected patients to follow changes over long periods of time, while CT and MRI are used to quantify changes in kidney parenchyma over shorter intervals [23]. In rodents, imaging accurately reflects kidney volumes, however, due to the small sizes of rodent kidneys, accurate evaluations of fractional cyst volume (FCV) are difficult and limited without the use of ultra-high field MRI (7 T and above) [25]. CT and MRI are the routine modalities used to evaluate the interventional efficacy of newly developed drugs in humans. MRI has not been used to evaluate ADPKD in cats, thus, baseline imaging studies could support the cats' role for evaluating therapeutics.

Three imaging modalities, US, CT, and MRI, are used to examine variation in disease presentation and disease progression in feline ADPKD. Imaging was compared to well-known biomarkers for CKD, such as urine specific gravity (USG), blood urea nitrogen (BUN), serum creatinine (sCr), glomerular filtration rate (GFR) and symmetric dimethylarginine (SDMA), which is a novel biomarker for feline CKD at the earlier stage [26]. Total kidney volume (TKV), total cystic volume (TCV) and FCV were determined for the first time in ADPKD cats, demonstrating the wide variation in disease presentation, the potential identification of rapid and slow progression individual cats and the potential to evaluate therapeutic interventions.

\section{Methods}

\section{Subjects}

The cats represented an ADPKD cat colony housed at the University of Missouri (MU), which has been maintained for over 20 years. Five Persian cats with ADPKD were originally donated by private owners to establish the colony. All animal procedures were conducted in accordance with the National Research Council Guide for the Care and Use of Laboratory Animals and were approved by the MU Animal Care and Use Committee (protocol \#8787). Cat housing and husbandry was overseen by MU Office of Animal Research. No cats were euthanized as part of this study. However, cats were euthanized by barbiturate overdose after sedation as dictated by poor health (renal failure). All cats were genotyped for the PKD1 c. $10063 \mathrm{C}>\mathrm{A}$ that causes feline ADPKD (data not shown) [17, 27]. Diagnostic imaging studies were performed at MU during normal clinic hours (8:00 am - 6:00 pm) from November 2016 to May 2017, for the initial imaging, and June 2018 for the follow-up CT and MRI imaging of two cats. Cats were fasted for at least $12 \mathrm{~h}$ prior to diagnostic imaging. At the time of sedation for imaging, blood was collected by 
jugular venipuncture for a complete blood count, serum chemistry including BUN, sCr, SDMA. Urine was collected for urinalysis via cystocentesis using ultrasound guidance.

\section{Ultrasonography}

US examinations were performed using an $8 \mathrm{MHz}$ micro-convex transducer on a dedicated ultrasound unit (Logiq 9, GE Healthcare, Wauwatosa, WI, USA). Each kidney was scanned in sagittal and transverse planes by either a first-year veterinary radiology resident (K.L.S.) or a board-certified veterinary radiologist (J.S.M.). The length was measured, using internal digital calipers, as the longest point between the cranial and caudal poles in the sagittal plane. Width and height were measured in the transverse plane. TKV was calculated using the prolate ellipsoid formula [28].

\section{Glomerular filtration rate}

GFRs were determined on the same day as the US imaging. Approximately $3 \mathrm{mCi}$ of ${ }^{99} \mathrm{mTc}$-diethylenetriaminepentaacetic acid was administered intravenously. Images were obtained using a gamma camera (Equistand II, Diagnostic Services, Middlesex, NJ.) with a low energy allpurpose collimator and using a $256 \times 256$ matrix. The GFR was calculated for each kidney by a single observer (K.L.S.), using Mirage software and a previously described method for scintigraphic uptake [29].

\section{Magnetic resonance imaging}

MRI was performed using a $3 \mathrm{~T}$ unit (Vantage Titan $3 \mathrm{~T}$, Canon Medical Systems, Tustin, CA, USA) and a transmit/receive coil. Scans obtained included dorsal T2 (TR 2469-5675, TE 120) weighted imaging (T2WI) with 2 $\mathrm{mm}$ slices, a $0.2 \mathrm{~mm}$ interspace gap, matrix $=320 \times 320$ 352 , and number of acquisitions $=1-3$. The scans were performed using respiratory gating to eliminate respiratory motion.

\section{Computed tomography}

CT scans were performed using a third generation 64 slice instrument (Aquillion 64, Canon Medical Systems, Tustin, CA, USA) under the same anesthetic event as the MRI scan. Follow-up imaging was performed for two cats at 12 months and 15 months, which were thought to have fast and slow cyst progression (i.e., high and low $\mathrm{FCV}$ at the youngest age), respectively.

Calculation of imaging parameters from CT and MRI data TKVs were calculated from US as described above. TKVs as determined by CT and MRI was measured using a planimetry method with Fiji software common to veterinary practice [30]. To determine the area per slice, the outline of the kidney was traced by free-hand by a single investigator (K.L.S.) on each contiguous slice. Total volumes of the kidneys were obtained by summing the areas of the slices and multiplying by slice thickness. TKV and TCV were also calculated by a single analyst (M.E.E.) using the minimal interaction rapid organ segmentation (MIROS) method [31]. Cyst progression rate was predicted from the FCV per months (age) and from follow-up CT and MRI for two cats at 12 months and 15 months as described above.

\section{Statistical analysis}

Means and standard deviations were calculated for 11 ADPKD cats for variables imaging indexes. All statistical analyses were performed using $\mathrm{R}$ software (version 3.3.3; R Foundation for Statistical Computing, Vienna, Austria). Comparisons of kidney volumes between male and female cats were analyzed using Mann-Whitney $U$ test. The Spearman's rank correlation test was used for the correlation analyses of imaging parameters and clinical data such as age, body weight and serum biomarkers. Kendall's coefficient of concordance was calculated to evaluate the reliability between or among each modality. High agreement is indicated when Kendall's coefficient of concordance (W) is higher than 0.75 . $P$ values $<0.05$ were considered statistically significant. Data are expressed as mean \pm standard deviation (SD).

\section{Results}

\section{Signalment and renal biomarkers}

Thirteen cats were examined, 11 cats were positive for the feline PKD1 variant (c.10063C > A) (Table 1). The two normal cats were one male (17.88 months, $4.9 \mathrm{~kg})$ and one female (15.12 months, $3.0 \mathrm{~kg})$. All 13 cats had normal physical examinations at the time of imaging, including normal kidneys determined by palpation. A majority of cats were within normal limits for biomarkers, including USG, BUN, sCr, SDMA, and GFR (Table 1). Expected correlations were identified within the serum biomarkers including a positive correlation for SDMA and $\mathrm{sCr}(r=0.88, p<0.001)$, a positive correlation for $\mathrm{sCr}$ with BUN $(r=0.78, p=0.004)$, and a positive correlation for SDMA with BUN $(r=0.61, p=0.047)$.

The oldest ADPKD cat (female; 8.1 years, Case 2 ) had an SDMA level of $15 \mu \mathrm{g} / \mathrm{dL}$, sCr level of $1.6 \mathrm{mg} / \mathrm{dL}$, and the lowest USG of 1.028. This cat was categorized as feline CKD Stage 2 based on the guidelines for feline CKD (International Renal interest Society; IRIS staging of CKD modified 2016; http://www.iris-kidney.com/guidelines/staging.html, accessed May 13, 2018). This cat was re-evaluated after 15 months and had mildly elevated levels of $\mathrm{sCr}(2.4 \mathrm{mg} / \mathrm{dL})$ and BUN $(36 \mathrm{mg} / \mathrm{dL})$ and lower USG (1.015) indicating advancing renal failure. At 19 months after the initial imaging, this cat rapidly declined within 2 weeks, reducing from normal weight of $3.9 \mathrm{~kg}$ 
Table 1 Signalment and renal biomarker data of ADPKD and control cats

\begin{tabular}{|c|c|c|c|c|c|c|c|c|}
\hline Cat ID & $\operatorname{Age}^{a}(\mathrm{mo})$ & Weight (kg) & Sex & SDMA (0-14) & $\mathrm{sCr}(0.5-2.2)$ & BUN (19-35) & USG $(1.035<)$ & GFR $(2.5<)$ \\
\hline Case 1 & 46.44 & 4.3 & $M$ & 9 & 1.1 & 16 & 1.051 & 2.3 \\
\hline Case 2 & 97.08 & 3.9 & $\mathrm{~F}$ & 15 & 1.6 & 24 & 1.028 & 2.78 \\
\hline Case $2^{\mathrm{b}}$ & $\sim 112$ & 3.9 & $\mathrm{~F}$ & NA & 2.4 & 36 & 1.015 & NA \\
\hline Case $2^{b}$ & $\sim 116$ & 2.7 & $\mathrm{~F}$ & NA & 11.9 & 260 & NA & NA \\
\hline Case 3 & 19.44 & 3.6 & $\mathrm{~F}$ & 12 & 1.2 & 24 & 1.055 & 3.95 \\
\hline Case $3^{b}$ & $\sim 32$ & $\sim 3.6$ & $\mathrm{~F}$ & 14 & 1.3 & 24 & 1.046 & NA \\
\hline Case 4 & 33.72 & 3.4 & $\mathrm{~F}$ & 9 & 1.2 & 22 & 1.06 & 2.6 \\
\hline Case 5 & 46.08 & 5.5 & M & 12 & 1.2 & 19 & 1.062 & 2.18 \\
\hline Case 6 & 47.04 & 4.2 & M & 9 & 0.9 & 13 & 1.06 & 3.12 \\
\hline Case $6^{b}$ & $\sim 63$ & $\sim 4.2$ & M & 8 & 0.7 & 18 & 1.062 & \\
\hline Case 7 & 33.36 & 5.2 & $\mathrm{~F}$ & 9 & 1 & 18 & 1.064 & 2.15 \\
\hline Case 8 & 22.92 & 6.0 & M & 8 & 0.9 & 14 & 1.06 & 2.83 \\
\hline Case 9 & 58.68 & 4.7 & M & 4 & 0.9 & 16 & 1.066 & 3.3 \\
\hline Case 10 & 15.12 & 4.1 & $\mathrm{~F}$ & 8 & 0.9 & 21 & 1.08 & 3.28 \\
\hline Case 11 & 16.44 & 4.8 & M & 8 & 0.9 & 13 & 1.056 & 2.64 \\
\hline mean $\pm S D$ & $39.67 \pm 23.89$ & $4.52 \pm 0.81$ & $6 F: 7 M$ & $9.36 \pm 2.84$ & $1.07 \pm 0.22$ & $18.18 \pm 4.14$ & $1.058 \pm 0.013$ & $2.83 \pm 0.55$ \\
\hline Control 1 & 15.12 & 3 & M & 4 & 0.9 & 17 & 1.063 & 2.66 \\
\hline Control 2 & 17.88 & 4.86 & $\mathrm{~F}$ & 8 & 0.9 & 24 & 1.066 & 2.97 \\
\hline
\end{tabular}

${ }^{\mathrm{a}}$ Age at time of imaging. M Male, F Female, SDMA Symmetric dimethylarginine (ug/dL), sCr serum creatinine (mg/dL), BUN Blood urea nitrogen (mg/dL), USG Urine specific gravity, GFR Glomerular filtration rate $(\mathrm{ml} / \mathrm{min} / \mathrm{kg}), N A$ Not available. ${ }^{b}$ Follow-up values were not used to calculate means \pm SD

to $2.7 \mathrm{~kg}$ and was euthanized due to end stage renal disease. All other APDKD cats had cysts and were classified as feline CKD Stage 1.

\section{Glomerular flow rate}

The two normal cats had GFRs of 2.66 and $2.97 \mathrm{ml} / \mathrm{min} /$ $\mathrm{kg}$. The majority of ADPKD affected cats had normal average GFRs, ranging from $2.15-3.95 \mathrm{ml} / \mathrm{min} / \mathrm{kg}$. Three cats showed below normal GFRs of 2.30, 2.18 and 2.15 $\mathrm{ml} / \mathrm{min} / \mathrm{kg}$ (Table 1 ). GFR had no suggested correlations with any biomarkers. GFR was not correlated with the TKV, regardless of modality used or the method of estimation of TKV (Additional file 1: Table S1). In addition, no correlation was identified between GFR and age of the cats $(r=0.1, p=0.78)$ (Additional file 1: Figure S1).

\section{Imaging}

The normal cats did not have incidental cysts. A majority of cysts in the ADPKD cats were located in the cortex or at the corticomedullary junction, however, some cysts were located in the medulla, which is consistent with previous reports for feline ADPKD [32]. All ADPKD cats had bilateral, multiple cysts but with variation in number and size (Fig. 1). Simple cysts were homogeneously hyperintense on T2WI MRI, however, some cats showed some T2-hypointense foci (Fig. 1).

\section{Kidney volume estimates}

Individual kidney volumes and TKVs were determined for each cat using three imaging modalities (Table 2, Additional file 1: Table S2). Males consistently had larger kidneys than females. The mean TKV ranged from $45.32 \pm 13.50 \mathrm{ml}$ for US to $69.39 \pm 12.08 \mathrm{ml}$ for planimetric MRI estimates. US-based volume estimates using the ellipsoid method were consistently the lowest estimated TKVs and MRI-based estimates were consistently the highest, especially the planimetric method for MRI. The TKV estimations for the two different methods were highly correlated within the CT and MRI modalities $(r=0.95-1, p<0.01)$. US estimates were more strongly correlated with CT (MIROS) $(r=0.84, p<0.01)$ than MRI (Additional file 1: Table S3). The ranks of the TKVs were consistent between planimetry and MIROS methods for MRI (W $=1, p=0.042$ ), and were fairly consistent between the planimetry and MIROS methods for CT (W= 0.92, $p=0.048$ ) (Additional file 1: Table S4). The four cats with the lowest kidney volumes, including controls, were ranked more consistently across modalities than the cats with the larger kidney volumes. The cats with the three largest TKVs were ranked consistently between US and both MRI methods, although the US estimates were $\sim 30 \%$ lower in volume. Approximately one-year post-imaging, TKVs for case 4 and case 5 were estimated by the water displacement 


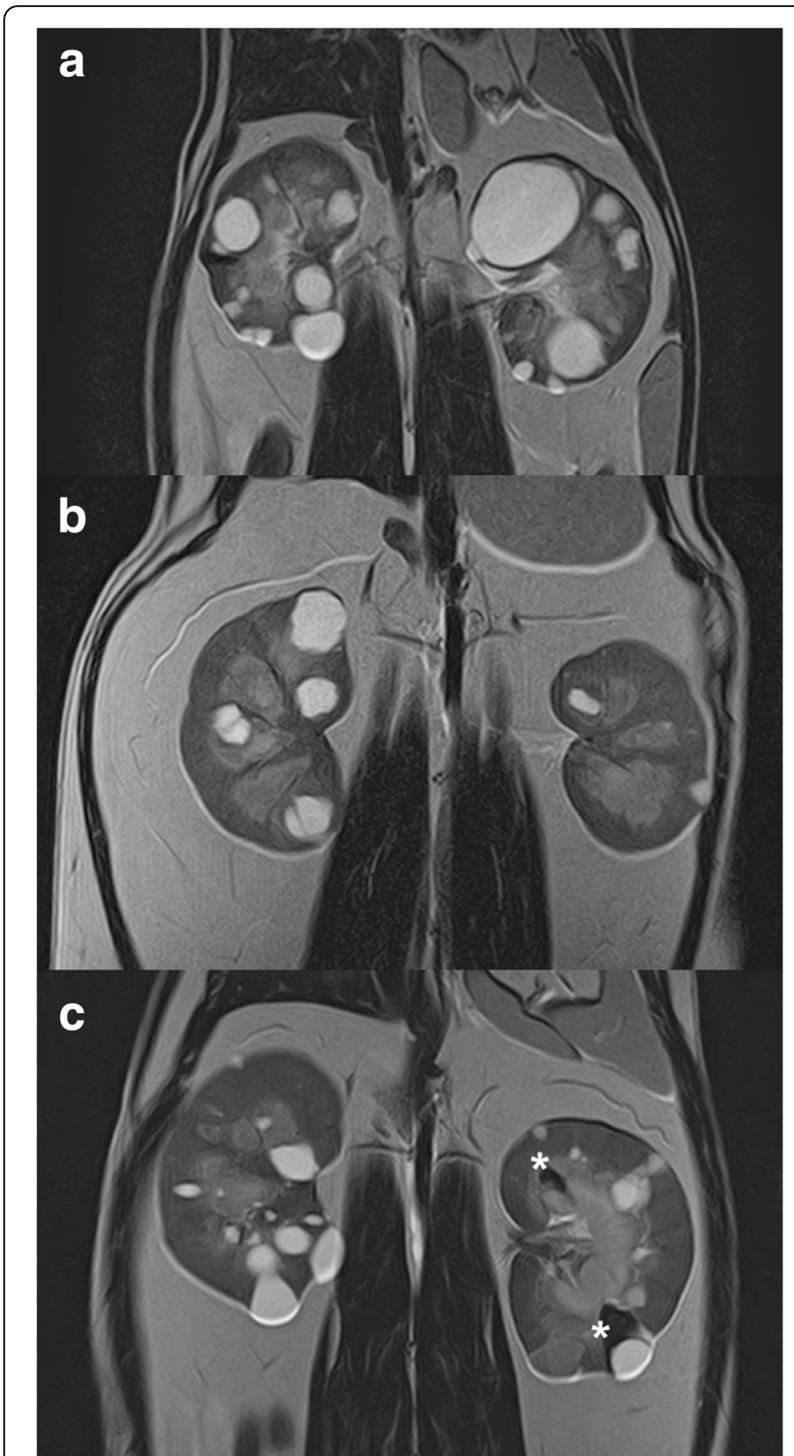

Fig. 1 T2-weighted MR imaging of ADPKD cats. Cysts in ADPKD positive cats were T2-hyperintense. a T2-weighted imaging of 1.6 years old cat (Case 3 ) that shows high FCV. b T2WI of 1.9 years old cat (Case 8) that shows low FCV. c T2-weighted imaging of 4.9 years old cat (Case 9). T2-hypointense foci are seen, suggesting the presence of T2-hypointense material such as hemorrhage. This finding was observed in several cats

method (data not shown) and were 26.5 and $51.2 \mathrm{ml}$, respectively (Additional file 1: Fig. S2).

\section{Cystic index}

TCVs were calculated using the MIROS method for both CT $(n=11)$ and MRI $(n=9)$ (Table 3, Additional file 1: Table S3). CT-based TCV ranged from 0.16 to $16.30 \mathrm{ml}$ $($ mean $=5.79 \pm 5.29 \mathrm{ml})$. MRI-based TCV ranged from 3.12 to $22.86 \mathrm{ml}($ mean $=9.83 \pm 7.39 \mathrm{ml}), \sim 70 \%$ larger than CT-based estimates. CT-based FCV ranged 0.63 to $28.22 \%$ (mean $=9.33 \pm 8.37 \%$ ). MRI-based FCV ranged from 6.18 to $44.64 \%$ (mean $=16.37 \pm 14.06 \%), ~ 75 \%$ larger than the CT estimates. Differences between right and left kidney FCV ranged from 0.03 to $12.32 \%$ based on CT imaging and from 0.71 to $17.29 \%$ for MRI (Table 3 ). The average difference in FCV between left and right kidneys was $4.57 \%$ for CT-based estimates and $6.96 \%$ for MRI-based estimates. In comparisons to biomarkers, concentrations of $\mathrm{sCr}$ were mildly associated with $\mathrm{FCVs}$ (CT- or MRI-based) ( $r=0.67, p=0.02 ; r=0.65, p=0.06$, respectively) and TCV (MRI-based) $(r=0.67, p=0.05)$. Additionally, concentration of BUN was mildly associated with MRI-based TCV $(r=0.77, p=0.02)$. GFR was not significantly correlated with TKV, TCV or FCV (Additional file 1: Table S1).

\section{TKV, TCV and FCV progression}

FCV increased approximately 0.04 to $1.08 \%$ per month based on CT imaging and 0.16 to $1.84 \%$ per month based on MRI. The scatter plots for cat age versus FCV suggests cystic growth rate is linear during CKD Stage 1 of disease in cats (Fig. 2). However, one cat was a suggested "outlier" for cystic volume as this young female cat (Case 3, 19.45 months) had the second largest TCV and FCV (MRI and CT), or also, could also be considered as the high end of a range in disease progression. Removal of this "outlier" cat improved correlations $(r>$ $0.8, p<0.05)$ for both MRI-based and CT-based TCV and FCV estimates (Fig. 2).

The general cyst progression rate (FCV per month) was an average of $0.19 \%$ per month (CT-based), if progression is considered linear, and $0.29 \%$ per month (MRI-based), when excluding the fastest outlier. The fast progression "outlier" cat (Case 3) had an expected FCV per month of $1.08 \%$ by CT and $1.84 \%$ by MRI. Case 6 was a slowly progressing cat with an expected FCV per month of $0.10 \%$ by CT and $0.16 \%$ by MRI. These two cats were re-evaluated after 12.6 and 15.0 months, respectively (Fig. 3, Table 4). TKV and TCV increased more drastically in the fast progression cat versus the slow progression cat and FCV increases were within the predicted estimates. However, the FCV increase based on MRI was counter intuitive as the estimate was $78.1 \%$ in the slow progression cat and only $33.62 \%$ for the fast progression cat. Also, the percent increase of FCV was similar between the two cats, 47.69 and $47.19 \%$ as estimated by CT. Based on the follow-up MRI (MIROS method), case 6 with the lowest FCV did not have a drastic increase in TKV (from 62.85 to $64.36 \mathrm{ml}$ ), however, had an $82.4 \%$ increase in TCV $(4.71-8.59 \mathrm{ml})$ and a $78.1 \%$ increase of FCV (7.49-13.35\%). Interestingly, percentage of monthly increase during the follow-up interval are within $9.8-11.9 \%$ in the both of cats, based on the both CT and MRI-based measurements. 
Table 2 Summary of ADPKD cat individual and total kidney volumes

\begin{tabular}{|c|c|c|c|c|c|c|c|c|}
\hline \multirow[t]{2}{*}{ Volume } & \multirow{2}{*}{$\begin{array}{l}\text { Modality } \\
\text { (method) }\end{array}$} & \multicolumn{2}{|c|}{ All $(n=11)^{*}$} & \multicolumn{2}{|c|}{ Male $(n=6)$} & \multicolumn{2}{|c|}{ Female $(n=5)^{*}$} & \multirow[t]{2}{*}{$P$-value } \\
\hline & & Mean & SD & Mean & SD & Mean & SD & \\
\hline \multirow[t]{5}{*}{ TKV } & US (Ellipsoid) & 45.32 & 13.50 & 51.49 & 6.87 & 37.92 & 16.46 & 0.2222 \\
\hline & CT (Planimetry) & 56.52 & 17.79 & 66.63 & 9.72 & 44.38 & 18.33 & 0.0303 \\
\hline & CT (MIROS) & 58.83 & 19.03 & 69.37 & 11.70 & 46.17 & 19.16 & 0.1255 \\
\hline & MRI (Planimetry) & 69.37 & 11.30 & 71.06 & 12.99 & 66.00 & 7.96 & NA \\
\hline & MRI (MIROS) & 62.91 & 10.36 & 64.71 & 11.41 & 59.32 & 8.63 & NA \\
\hline \multirow[t]{5}{*}{ RKV } & US (Ellipsoid) & 21.91 & 6.93 & 25.72 & 4.64 & 17.33 & 6.72 & 0.08225 \\
\hline & CT (Planimetry) & 27.54 & 9.87 & 34.07 & 6.06 & 19.71 & 7.55 & 0.008658 \\
\hline & CT (MIROS) & 28.59 & 10.56 & 35.27 & 7.44 & 20.57 & 7.89 & 0.01732 \\
\hline & MRI (Planimetry) & 34.67 & 7.81 & 36.90 & 7.65 & 30.21 & 7.28 & NA \\
\hline & MRI (MIROS) & 30.89 & 7.08 & 33.35 & 6.71 & 25.96 & 5.79 & NA \\
\hline \multirow[t]{5}{*}{ LKV } & US (Ellipsoid) & 23.41 & 7.15 & 25.77 & 2.95 & 20.59 & 9.93 & 0.9307 \\
\hline & CT (Planimetry) & 28.97 & 8.82 & 32.56 & 4.52 & 24.67 & 11.25 & 0.329 \\
\hline & CT (MIROS) & 30.24 & 9.40 & 34.10 & 5.15 & 25.61 & 11.77 & 0.1775 \\
\hline & MRI (Planimetry) & 34.71 & 4.99 & 34.16 & 6.14 & 35.80 & 1.66 & NA \\
\hline & MRI (MIROS) & 32.03 & 4.57 & 33.35 & 25.56 & 33.36 & 32.79 & NA \\
\hline
\end{tabular}

TKV Total kidney volume, RKV Right kidney volume, LKV Left kidney volume, NA Not applicable. *all $n=9$ for MRI, female $n=3$ for MRI.

Each estimate of TCV, FCV, and FCV per month were significantly correlated between modalities (i.e., CT-based and MRI-based) $(r=0.95-0.98, p<0.01)$ (Additional file 1: Table S3). The rank of TCV was the same between the modalities except for one cat. Kendall's coefficient of concordance showed $\mathrm{W}=0.89(p=0.075)$. The rank of FCV showed $\mathrm{W}=0.82(p=0.11)$ with Kendall's coefficient of concordance (Additional file 1: Table S4).

\section{Discussion}

ADPKD is caused by PKD1 variants and is a common genetic and life-threatening disease for both humans and domestic cats. Although a variety of rodent models support PKD research, none support long-term trials of therapeutics. ADPKD domestic cats have a stop codon in exon 46 , disrupting $\sim 30 \%$ of polycystin-1 [17]. The disease is autosomal dominant, the homozygous state is

Table 3 TCV and FCV estimated from CT and MRI in ADPKD cats

\begin{tabular}{|c|c|c|c|c|c|c|c|c|c|c|c|c|c|c|c|c|c|c|c|}
\hline \multirow[b]{3}{*}{ Cat ID } & \multirow[b]{3}{*}{$\operatorname{Age}^{a}(\mathrm{mo})$} & \multicolumn{9}{|l|}{$\mathrm{CT}$} & \multicolumn{9}{|l|}{ MRI } \\
\hline & & \multicolumn{3}{|l|}{$\overline{\mathrm{TCV}}$} & \multicolumn{3}{|c|}{ FCV (\%) } & \multicolumn{3}{|c|}{ FCV/Mo (\%) } & \multicolumn{3}{|l|}{$\overline{\mathrm{TCV}}$} & \multicolumn{3}{|c|}{ FCV (\%) } & \multicolumn{3}{|c|}{ FCV/Mo (\%) } \\
\hline & & Right & Left & Total & Right & Left & Total & Right & Left & Total & Right & Left & Total & Right & Left & Total & Right & Left & Total \\
\hline Case 1 & 46.44 & 1.82 & 1.94 & 3.76 & 6.00 & 7.66 & 6.75 & 0.13 & 0.16 & 0.15 & 3.35 & 2.94 & 6.29 & 11.36 & 12.07 & 11.68 & 0.24 & 0.26 & 0.25 \\
\hline Case 2 & 97.08 & 7.55 & 8.75 & 16.30 & 33.65 & 24.76 & 28.22 & 0.35 & 0.26 & 0.29 & 10.04 & 12.82 & 22.86 & 49.26 & 41.58 & 44.64 & 0.51 & 0.43 & 0.46 \\
\hline Case 3 & 19.44 & 3.49 & 9.13 & 12.63 & 13.93 & 26.25 & 21.09 & 0.72 & 1.35 & 1.08 & 6.68 & 14.24 & 20.92 & 26.13 & 43.43 & 35.85 & 1.34 & 2.23 & 1.84 \\
\hline Case 4 & 33.72 & 0.91 & 0.90 & 1.81 & 7.09 & 7.18 & 7.13 & 0.21 & 0.21 & 0.21 & NA & & & & & & & & \\
\hline Case 5 & 46.08 & 1.82 & 3.66 & 5.48 & 4.91 & 10.05 & 7.46 & 0.11 & 0.22 & 0.16 & 2.86 & 4.77 & 7.63 & 8.29 & 14.54 & 11.34 & 0.18 & 0.32 & 0.25 \\
\hline Case 6 & 47.04 & 0.29 & 2.52 & 2.82 & 1.18 & 7.74 & 4.91 & 0.03 & 0.16 & 0.10 & 0.72 & 3.99 & 4.71 & 2.47 & 11.82 & 7.49 & 0.05 & 0.25 & 0.16 \\
\hline Case 7 & 33.36 & 0.91 & 1.64 & 2.55 & 3.00 & 5.06 & 4.06 & 0.09 & 0.15 & 0.12 & 1.74 & 2.51 & 4.25 & 5.45 & 6.89 & 6.21 & 0.16 & 0.21 & 0.19 \\
\hline Case 8 & 22.92 & 2.87 & 0.37 & 3.24 & 7.53 & 1.09 & 4.50 & 0.33 & 0.05 & 0.20 & 5.23 & 0.69 & 5.92 & 13.09 & 2.05 & 8.05 & 0.57 & 0.09 & 0.35 \\
\hline Case 9 & 58.68 & 6.77 & 5.14 & 11.91 & 14.52 & 12.60 & 13.62 & 0.25 & 0.21 & 0.23 & 7.18 & 5.58 & 12.76 & 17.05 & 14.62 & 15.90 & 0.29 & 0.25 & 0.27 \\
\hline Case 10 & 15.12 & 0.07 & 0.08 & 0.16 & 0.61 & 0.64 & 0.63 & 0.04 & 0.04 & 0.04 & NA & & & & & & & & \\
\hline Case 11 & 16.44 & 0.60 & 2.43 & 3.03 & 1.71 & 6.82 & 4.30 & 0.10 & 0.41 & 0.26 & 0.73 & 2.39 & 3.12 & 2.92 & 9.38 & 6.18 & 0.18 & 0.57 & 0.38 \\
\hline Average & 39.67 & 2.46 & 3.32 & 5.79 & 8.56 & 9.99 & 9.33 & 0.21 & 0.29 & 0.26 & 4.28 & 5.55 & 9.83 & 15.11 & 17.28 & 16.37 & 0.39 & 0.51 & 0.46 \\
\hline SD & 23.89 & 2.55 & 3.13 & 5.29 & 9.55 & 8.42 & 8.37 & 0.20 & 0.36 & 0.28 & 3.22 & 4.76 & 7.39 & 14.82 & 14.78 & 14.06 & 0.39 & 0.66 & 0.53 \\
\hline
\end{tabular}

${ }^{\mathrm{a}}$ Age in months at the initial scan. NA: Not available 

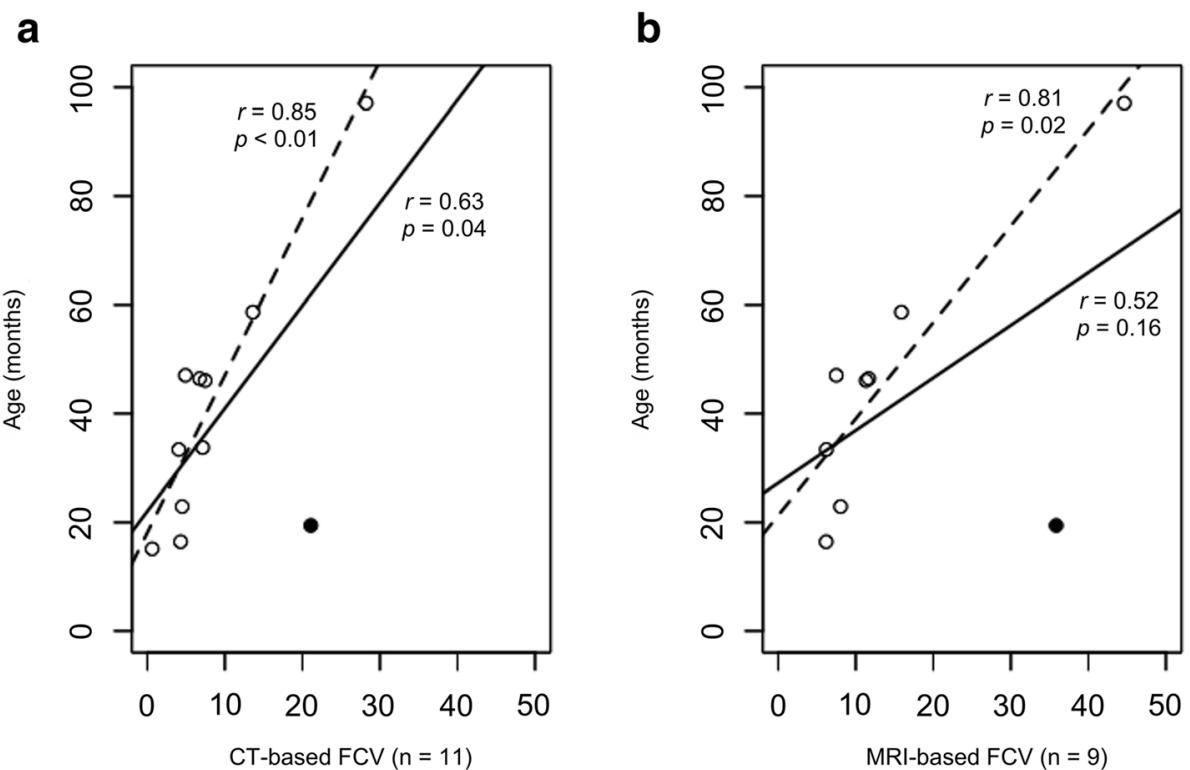

Fig. 2 FCV correlation with age in feline ADPKD. a The graph illustrates significant positive correlation between CT-based FCV and age amongst 11 ADPKD cats (solid line). One cat is a suggested outlier (bold dots). When excluding this cat, the correlation increases (dashed line). b The graph demonstrates strong positive correlation between MRI-based FCV and age among eight ADPKD cats when the one outlier was excluded

lethal in utero, and cysts develop prior 8 months of age. Thus, this cat models mimics the human condition genetically. However, little is known about the changes in kidney imaging parameters in ADPKD cats, especially TKV, TCV, and FCV.

The rate and extent of cystic progression has not been examined in cats, although generally, cysts worsen as the cat ages. The average life span of cats is $~ 13-17$ years of age and many die of CKD [33, 34]. One of the factors leading to the discovery of ADPKD was early CKD as cats were dying at 3-4 years old. However, many cats with ADPKD live a normal life span. Thus, the variation in disease severity and rate of progression is recognized but undocumented. Imaging is required to determine disease severity and to monitor progression even though a genetic test exists for feline ADPKD [17, 27]. The range in severity suggests additional genetic and nongenetic factors influence disease progression.

Eleven ADPKD cats were examined by different modalities to quantify the variation in the cats and to compare imaging modalities. Ten of eleven cats had no suggestion of renal compromise other than the presence of cysts, thus renal biomarkers, including SDMA, $\mathrm{sCr}$ and $\mathrm{BUN}$, are not predictive risk indicators of feline ADPKD and disease severity. GFR was also not indicative of kidney disease in the ADPKD cats. Significant parenchymal loss is likely to be required before abnormal GFRs are observed in APDKD cats [32]. The GFRs were within normal limits or low for the ADPKD cats, including the oldest cat and five ADPKD cats with $15 \%$ FCV. Three cats with lower FCV had mildly lowered GFRs, which may be partially due to reduced renal artery pressure secondary to the anesthesia. The age of the cat cohort is relatively young (mean: 39.7 months [range: 16.4-97.1 months]. Many human ADPKD patients have no obvious clinical symptoms until the third or fourth decade of life [35] and renal function usually remains normal until the fourth to sixth decade of life [36]. A recent study of 377 cats from Japan indicated of cats with the cat PKD1 mutation, the incidence of a high concentration of plasma Cre $(>1.6 \mathrm{mg} / \mathrm{dl}$ : $\geq$ IRIS-CKD stage 2) was greater in cats older than 3 years old, and especially in those older than 7 years. In contrast, a few cats aged $\geq 9$ years had low plasma Cre concentrations $(\leq 1.6 \mathrm{mg} / \mathrm{dl})$ [37]. Therefore, the correlation between TKV and GFR may improve in older ADPKD cats.

$\mathrm{TKV}$, rather than renal function, is suggested as the more appropriate biomarker for monitoring and predicting disease progression in human medicine [38]. In humans, TKV measurements are obtained by MRI or CT to assess the efficacy of therapeutic interventions $[23,39,40]$. US, CT and MRI are all minimally invasive and highly diagnostic for ADPKD in cats, with US being the most rapid, least expensive, and most accessible. MRI T2WI is sensitive and sufficient for volume measurement and $\mathrm{CT}$ is associated with radiation exposure, hence $\mathrm{CT}$ is less favored in human medicine $[2,40]$. However, CT is favored in cats due to decreased 


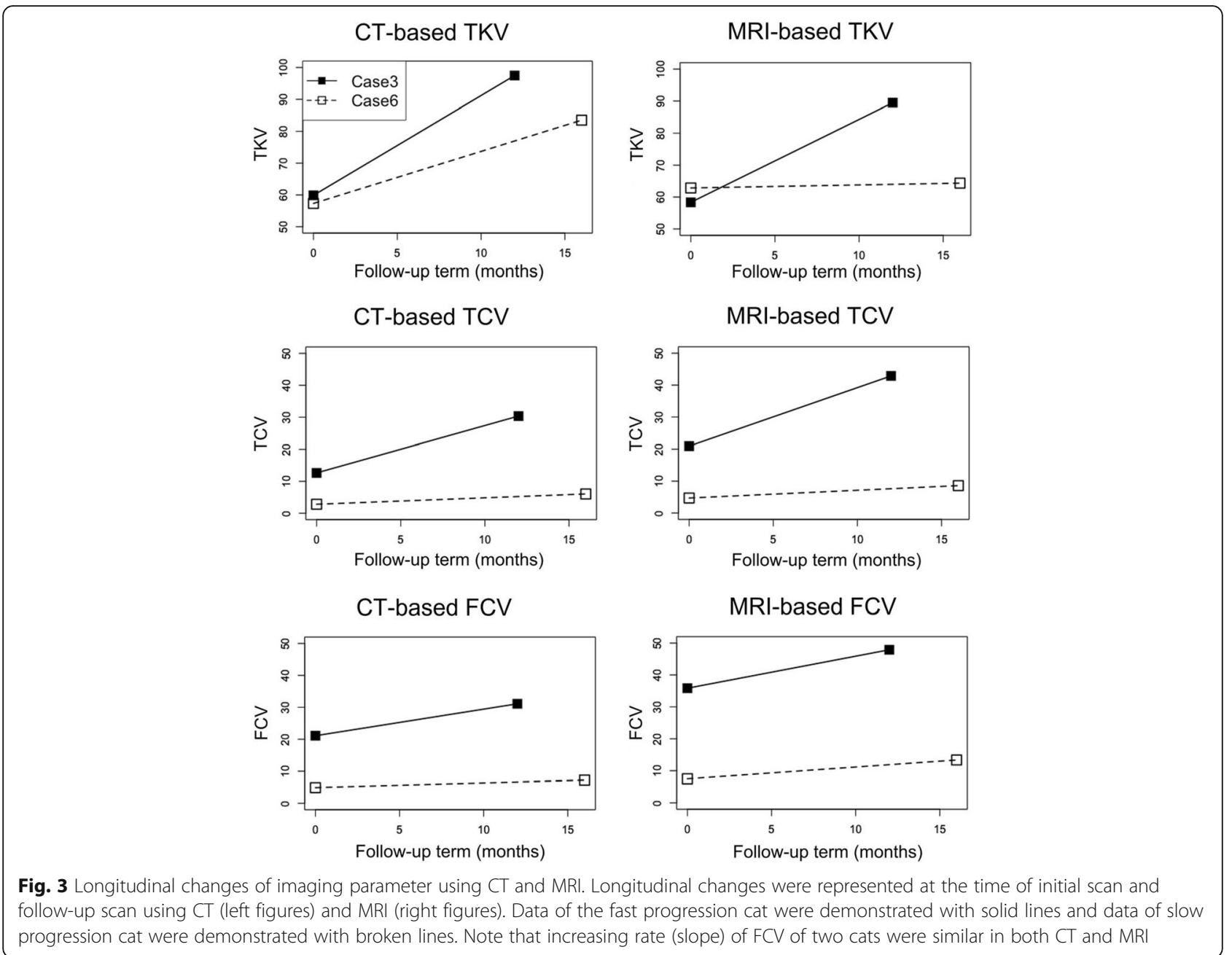

Table 4 Renal volumetric changes in cats with ADPKD

\begin{tabular}{|c|c|c|c|c|c|c|c|c|c|c|}
\hline Volume & Case & Modality & Initial & Follow up & Increase (ml) & Increase/mo. (ml) & Increase (\%) & Monthly increase (\%) & Initial FCV/mo & $\begin{array}{l}\text { Expected } \\
\text { increase }\end{array}$ \\
\hline \multirow[t]{4}{*}{ TKV } & Fast 3 & CT & 59.88 & 97.48 & 37.60 & 2.98 & 62.79 & 12.92 & & \\
\hline & & MRI & 58.35 & 89.49 & 31.14 & 2.47 & 53.37 & 12.78 & & \\
\hline & Slow 6 & CT & 57.33 & 83.52 & 26.19 & 1.75 & 45.68 & 9.71 & & \\
\hline & & MRI & 62.85 & 64.36 & 1.51 & 0.10 & 2.40 & 6.83 & & \\
\hline \multirow[t]{4}{*}{ TCV } & Fast 3 & CT & 12.63 & 30.36 & 17.73 & 1.41 & 140.43 & 19.08 & & \\
\hline & & MRI & 20.92 & 42.87 & 21.95 & 1.74 & 104.92 & 17.08 & & \\
\hline & Slow 6 & $C T$ & 2.82 & 6.04 & 3.22 & 0.21 & 114.43 & 14.30 & & \\
\hline & & MRI & 4.71 & 8.59 & 3.88 & 0.26 & 82.38 & 12.16 & & \\
\hline \multirow[t]{4}{*}{ FCV } & Fast 3 & CT & 21.09 & 31.14 & 10.06 & 0.80 & 47.69 & 11.72 & 1.08 & 13.61 \\
\hline & & $\mathrm{MRI}$ & 35.85 & 47.90 & 12.05 & 0.96 & 33.62 & 11.13 & 1.84 & 23.18 \\
\hline & Slow 6 & $C T$ & 4.91 & 7.23 & 2.32 & 0.15 & 47.19 & 9.81 & 0.10 & 1.5 \\
\hline & & MRI & 7.49 & 13.35 & 5.85 & 0.39 & 78.10 & 11.87 & 0.16 & 2.4 \\
\hline
\end{tabular}


anesthesia requirements and the minimal concern of long-term consequences of radiation exposure.

US, CT and MRI were conducted in ADPKD cats to estimate TKV. Normal cat kidney volumes have been estimated using water displacement at $18.99 \pm 7.68 \mathrm{~cm}^{3}$, and US and CT imaging from $14.8 \pm 2.9 \mathrm{ml}$ to $19.01 \pm$ $7.55 \mathrm{ml}$, respectively $[32,41]$. For ADPKD cats, total kidney volumes have been estimated as $27.4 \pm 10.3 \mathrm{ml}$ using US and $29.3 \pm 13.4 \mathrm{ml}$ by CT [32], $~ 30 \%$ larger than normal cat kidneys. In this study, the 11 ADPKD cats had kidney volume estimates from $23.0 \pm 6.91 \mathrm{ml}$ using US to as high as $34.69 \pm 6.36 \mathrm{ml}$ using the planimetric method for MRI thereby overlapping within normal limits but also have significantly larger TKVs. Overall, US and planimetry CT TKV estimates were slightly smaller, however, within the ranges of previous study. The cats in the previous study (mean age: $59 \pm 10$ months) [32] were an average of 20 months older than this study (mean age: $39.67 \pm 23.89$ months), thus would be expected to have larger TKVs. The TKV estimates for a given cat were increasing larger over US-based TKV estimates by $20,22,27,34 \%$, estimating by planimetry CT, MIROS CT, MIROS MRI, and planimetry MRI, respectively. The TKV for case 4 that was estimated by water displacement was consistent with CT estimates, but case 5 estimates were over-estimated by $\mathrm{CT}$ and MRI, potentially due to the adipose tissue in this overweight cat.

In one study, cyst growth in humans is reported as relatively symmetrically and at a steady rate [42]. However, other research has indicated variable expression of cyst progression, even in the same family [43, 44]. In the cats, FCVs were highly correlated with age, supporting cyst size increasing with age and a steady rate. However, Case 3 showed 1.08 and $1.84 \%$ increase in FCV per month, by CT and MRI, respectively, which is approximately six times the average increase than the other ADPKD cats. Once this "outlier" was removed from the correlation analysis, FCV per month showed an average of 0.19 and $0.29 \%$ by CT and MRI, respectively, and was more highly correlated with age. Thus, for cats, most cats have a consistent rate of disease progression, however, some cats are highly variability. Furthermore, the value on TCV and FCV showed wide ranges as shown in Table 3. Although ages were not uniform, these findings also indicated that variability of cyst progression speed among feline ADPKD cats, regardless of genetic homogeneity for the mutation. Feline ADPKD is individually variable between kidneys of a given cat, even though all cats had the same germline mutation (PKD1 c.10063C > A), suggesting other factors modify disease expression and progression.

Cats as a large animal model are clearly an asset to evaluate the efficacy and safety of the development of drugs and gene therapies [45, 46]. Although the exact same mutation is not found in cats and humans, cats and humans have similarly disruptive mutations that truncate approximately $30 \%$ of polycystin-1. Feline ADPKD may fill a void of translational research between rodent and human ADPKD. The rodent models do not perfectly recapitulate human ADPKD in terms of differences in lifespan, metabolism, and renal anatomy [47]. Feline ADPKD has the potential to overcome these differences. Although feline ADPKD is caused by a single PKD1 c.10063C > A, considering progression variability, disease progression of feline ADPKD is likely influenced by other genetic and/or environmental factors, such as those identified in humans [48-51]. In addition, cats could support studies focusing on pleiotrophic effects of ADPKD. A recent study showed co-occurrence of hepatic and renal cysts was found in 20 (12.6\%) out of 159 cat cases with renal cyst(s), and all cases were positive for the PKD1 mutation [37]. Identification of genetic modifiers could lead to selection of appropriate cats with ADPKD for therapeutic trials and reducing animal use and improving study design.

\section{Conclusions}

In conclusion, TKV, TCV, and FCV estimations are valuable for evaluating disease status of feline ADPKD and could lead to renal failure risk classifications analogous to humans [52, 53 ]. The current study shows imagingbased estimations for TKV, TCV, FCV, and FCV per month, can be easily determined for cats with ADPKD using CT and MRI. While MRI is preferred to CT in human medicine due to radiation exposure, CT is more practical to evaluate disease progression for feline ADPKD because of rapid image acquisition that only requires sedation or light anesthesia, lower cost, and increasing availability. This study also demonstrates that cystic progression is individual and variable in cats and since cats have only on ADPKD variant and the genetic model is highly similar to humans, cats should be useful for studies focused on genetic modifiers and efficacy of therapeutics.

\section{Additional file}

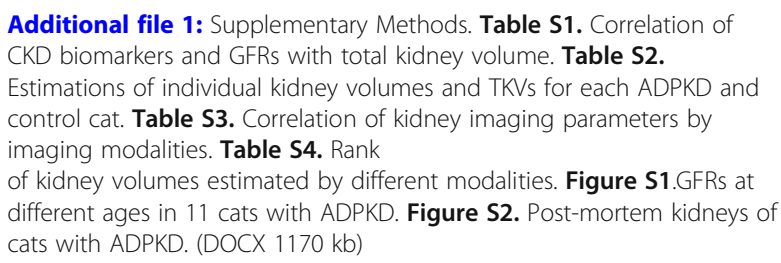

Additional file 1: Supplementary Methods. Table S1. Correlation of CKD biomarkers and GFRs with total kidney volume. Table S2. Estimations of individual kidney volumes and TKVs for each ADPKD and control cat. Table S3. Correlation of kidney imaging parameters by imaging modalities. Table S4. Rank of kidney volumes estimated by different modalities. Figure S1.GFRs at different ages in 11 cats with ADPKD. Figure S2. Post-mortem kidneys of cats with ADPKD. (DOCX $1170 \mathrm{~kb})$

\section{Abbreviations}

ADPKD: Autosomal dominant polycystic kidney disease; BUN: Blood urea nitrogen; CKD: Chronic kidney disease; CT: Computed tomography; FCV: Fractional cystic volume; GFR: Glomerular filtration rate; 
IRIS: International Renal Interest Society; LKV: Left kidney volume; MIROS: Minimal interaction rapid organ segmentation; MRI: Magnetic resonance imaging; mRNA: Messenger RNA; MU: University of Missouri; PKD: Polycystic kidney disease; PKD1: Polycystin 1; PKDB: Polycystic Kidney Disease Mutation Database; RKV: Right kidney volume; sCr: Serum creatinine; SD: Standard deviation; SDMA: Symmetric dimethylarginine; TCV: Total cystic volume; TKV: Total kidney volume; US: Ultrasonography; USG: Urine specific gravity

\section{Acknowledgements}

We appreciate the support of University of Missouri, Veterinary Medical Center Anesthesiology and Radiology support staff for assistance with this project. A part of this study was presented as a poster at the 2018 American College of Veterinary Internal Medicine (ACVIM) Forum, in Seattle, WA, USA.

\section{Authors' contributions}

LAL conceived and designed the study; KLS, MEE, JSM, and TLK obtained the data; $Y Y$ analyzed the data; $Y Y$ made the figures, $Y Y$ and $K L S$ wrote the paper, YY, LAL, KLS, TLK revised the paper; all authors approved the final version of the manuscript.'

\section{Funding}

Funding was provided by the Gilbreath McLorn Endowment (L.A.L), a CVM Clinical Scientist award (K.L.S and J.S.M), Overseas Challenge Program for Young Researchers, under the Japan Society for the Promotion of Science (Y.Y). Also supported in part by the Mayo Clinic Robert M. and Billie Kelley Pirnie Translational PKD Center and the NIDDK grants P30DK090728 (M.E.E and T.L.K) and K01DK110136 (T.L.K). The funders of the project did not have a role in study design nor interpretation of the data and did not influence the outcome of the study.

\section{Availability of data and materials}

All data generated or analyzed during this study are included in this published article [and its supplementary information files]. Raw data files of imaging are available from the corresponding author on reasonable request.

\section{Ethics approval and consent to participate}

All animal procedures were conducted in accord with the National Research Council Guide for the Care and Use of Laboratory Animals and were approved by the MU Animal Care and Use Committee (protocol \#8787).

\section{Consent for publication}

Not Applicable.

\section{Competing interests}

The authors declare that they have no competing interests.

\section{Author details}

${ }^{1}$ Department of Veterinary Medicine and Surgery, College of Veterinary Medicine, University of Missouri, Columbia, MO, USA. ${ }^{2}$ Laboratory of Veterinary Radiology, Nippon Veterinary and Life Science University, Tokyo, Japan. ${ }^{3}$ Division of Nephrology and Hypertension, Mayo Clinic, Rochester, MN, USA. ${ }^{4}$ Department of Radiology, Mayo Clinic, Rochester, MN, USA.

Received: 14 March 2019 Accepted: 1 July 2019

\section{Published online: 12 July 2019}

\section{References}

1. Harris PC, Torres VE. Polycystic kidney disease. Annu Rev Med. 2009;60: 321-37

2. Chapman AB, Devuyst O, Eckardt KU, et al. Autosomal-dominant polycystic kidney disease (ADPKD): executive summary from a kidney disease: improving global outcomes (KDIGO) controversies conference. Kidney Int. 2015;88(1):17-27.

3. Mikolajczyk AE, Te HS, Chapman AB. Gastrointestinal manifestations of autosomal-dominant polycystic kidney disease. Clin Gastroenterol Hepatol. 2017;15(1):17-24.

4. Spithoven EM, Kramer A, Meijer E, et al. Renal replacement therapy for autosomal dominant polycystic kidney disease (ADPKD) in Europe: prevalence and survival--an analysis of data from the ERA-EDTA registry. Nephrol Dial Transplant. 2014;29(Suppl 4):iv15-25.
5. Cornec-Le Gall E, Audrezet MP, Chen JM, et al. Type of PKD1 mutation influences renal outcome in ADPKD. J Am Soc Nephrol. 2013;24(6):1006-13.

6. Harris PC, Bae KT, Rossetti $S$, et al. Cyst number but not the rate of cystic growth is associated with the mutated gene in autosomal dominant polycystic kidney disease. J Am Soc Nephrol. 2006;17(11):3013-9.

7. Heyer CM, Sundsbak JL, Abebe KZ, et al. Predicted mutation strength of nontruncating PKD1 mutations aids genotype-phenotype correlations in autosomal dominant polycystic kidney disease. J Am Soc Nephrol. 2016; 27(9):2872-84

8. Autosomal Dominant Polycystic Kidney Disease Mutation Database: PKDB [http://pkdb.pkdcure.org]. Accessed 13 May 2018.

9. Hughes J, Ward CJ, Peral B, et al. The polycystic kidney disease 1 (PKD1) gene encodes a novel protein with multiple cell recognition domains. Nat Genet. 1995;10(2):151-60.

10. Kirsch S, Pasantes J, Wolf A, et al. Chromosomal evolution of the PKD1 gene family in primates. BMC Evol Biol. 2008;8:263.

11. Germino GG. Autosomal dominant polycystic kidney disease: a two-hit model. Hosp Pract (1995). 1997;32(3):81-2 85-88, 91-82 passim.

12. Barrs VR, Gunew M, Foster SF, et al. Prevalence of autosomal dominant polycystic kidney disease in Persian cats and related-breeds in Sydney and Brisbane. Aust Vet J. 2001;79(4):257-9.

13. Barthez PY, Rivier P, Begon D. Prevalence of polycystic kidney disease in Persian and Persian related cats in France. J Feline Med Surg. 2003;5(6): 345-7.

14. Biller DS, DiBartola SP, Eaton KA, et al. Inheritance of polycystic kidney disease in Persian cats. J Hered. 1996;87(1):1-5.

15. Cannon MJ, MacKay AD, Barr FJ, et al. Prevalence of polycystic kidney disease in Persian cats in the United Kingdom. Vet Rec. 2001;149(14):409-11.

16. Eaton KA, Biller DS, DiBartola SP, et al. Autosomal dominant polycystic kidney disease in Persian and Persian-cross cats. Vet Pathol. 1997;34(2): $117-26$.

17. Lyons LA, Biller DS, Erdman CA, et al. Feline polycystic kidney disease mutation identified in PKD1. J Am Soc Nephrol. 2004;15(10):2548-55.

18. Lee YJ, Chen HY, Hsu WL, et al. Diagnosis of feline polycystic kidney disease by a combination of ultrasonographic examination and PKD1 gene analysis. Vet Rec. 2010;167(16):614-8.

19. Nivy R, Lyons LA, Aroch I, Segev G. Polycystic kidney disease in four British shorthair cats with successful treatment of bacterial cyst infection. J Small Anim Pract. 2015;56(9):585-9.

20. Bonazzi M, Volta A, Gnudi G, et al. Prevalence of the polycystic kidney disease and renal and urinary bladder ultrasonographic abnormalities in Persian and exotic shorthair cats in Italy. J Feline Med Surg. 2007;9(5): 387-91.

21. Pedersen KM, Pedersen HD, Haggstrom J, et al. Increased mean arterial pressure and aldosterone-to-renin ratio in Persian cats with polycystic kidney disease. J Vet Intern Med. 2003;17(1):21-7.

22. Littman MP. Genetic basis for urinary tract diseases. In: Elliott J, Grauer GF, Westropp JL, editors. BSAVA manual of canine and feline nephrology and urology. Edn. Gloucester: British Small Animal Veterinary Association; 2017. p. $172-84$.

23. Bae KT, Grantham JJ. Imaging for the prognosis of autosomal dominant polycystic kidney disease. Nat Rev Nephrol. 2010;6(2):96-106.

24. Wills SJ, Barrett EL, Barr FJ, et al. Evaluation of the repeatability of ultrasound scanning for detection of feline polycystic kidney disease. J Feline Med Surg. 2009:11(12):993-6.

25. Irazabal MV, Mishra PK, Torres VE, Macura SI. Use of ultra-high field MRI in small rodent models of polycystic kidney disease for in vivo phenotyping and drug monitoring. J Vis Exp. 2015;100:e52757.

26. Hall JA, Yerramilli $M$, Obare $E$, et al. Comparison of serum concentrations of symmetric dimethylarginine and creatinine as kidney function biomarkers in cats with chronic kidney disease. J Vet Intern Med. 2014;28(6):1676-83.

27. Grahn RA, Biller DS, Young AE, et al. Genetic testing for feline polycystic kidney disease. Anim Genet. 2004;35(6):503-4.

28. Nyland TG, Fisher PE, Gregory CR, Wisner ER. Ultrasonographic evaluation of renal size in dogs with acute allograft rejection. Vet Radiol Ultrasound. 1997; 38(1):55-61

29. Daniel GB, Mitchell SK, Mawby D, et al. Renal nuclear medicine: a review. Vet Radiol Ultrasound. 1999;40(6):572-87.

30. Schindelin J, Arganda-Carreras I, Frise E, et al. Fiji: an open-source platform for biological-image analysis. Nat Methods. 2012;9(7):676-82. 
31. Kline $T L$, Edwards ME, Korfiatis P, et al. Semiautomated segmentation of polycystic kidneys in T2-weighted MR images. Am J Roentgen. 2016;207(3): 605-13.

32. Reichle JK, DiBartola SP, Leveille R. Renal ultrasonographic and computed tomographic appearance, volume, and function of cats with autosomal dominant polycystic kidney disease. Vet Radiol Ultrasound. 2002;43(4):368-73.

33. Cozzi B, Ballarin C, Mantovani R, Rota A. Aging and veterinary Care of Cats, dogs, and horses through the Records of Three University Veterinary Hospitals. Front Vet Sci. 2017:4:14.

34. O'Neill DG, Church DB, McGreevy PD, et al. Longevity and mortality of cats attending primary care veterinary practices in England. J Feline Med Surg. 2015;17(2):125-33.

35. Grantham JJ. Clinical practice. Autosomal dominant polycystic kidney disease. N Engl J Med. 2008;359(14):1477-85.

36. Torres VE, Harris PC, Pirson Y. Autosomal dominant polycystic kidney disease. Lancet. 2007;369(9569):1287-301.

37. Sato R, Uchida N, Kawana Y, et al. Epidemiological evaluation of cats associated with feline polycystic kidney disease caused by the feline PKD1 genetic mutation in Japan. J Vet Med Sci. 2019. https://doi.org/10.1292/ jvms.18-0309.

38. Alam A, Dahl NK, Lipschutz JH, et al. Total kidney volume in autosomal dominant polycystic kidney disease: a biomarker of disease progression and therapeutic efficacy. Am J Kidney Dis. 2015;66(4):564-76.

39. Torres VE, Higashihara E, Devuyst O, et al. Effect of Tolvaptan in autosomal dominant polycystic kidney disease by CKD stage: results from the TEMPO 3:4 trial. Clin J Am Soc Nephrol. 2016;11(5):803-11.

40. Mai J, Lee WW, Lopez-Vargas P, et al. KHA-CARI autosomal dominant polycystic kidney disease guideline: monitoring disease progression. Semin Nephrol. 2015;35(6):565-571.e18.

41. Tyson R, Logsdon SA, Werre SR, Daniel GB. Estimation of feline renal volume using computed tomography and ultrasound. Vet Radiol Ultrasound. 2013; 54(2):127-32.

42. Grantham JJ, Torres VE, Chapman AB, et al. Volume Progression in Polycystic Kidney Disease. N Engl J Med. 2006;354(20):2122-30.

43. Milutinovic J, Rust PF, Fialkow PJ, et al. Intrafamilial phenotypic expression of autosomal dominant polycystic kidney disease. Am J Kidney Dis. 1992; 19(5):465-72.

44. Rossetti S, Burton S, Strmecki L, et al. The position of the polycystic kidney disease 1 (PKD1) gene mutation correlates with the severityof renal disease. J Am Soc Nephrol. 2002;13(5):1230-7.

45. Vite $\mathrm{CH}$, Bagel JH, Swain GP, et al. Intracisternal cyclodextrin prevents cerebellar dysfunction and Purkinje cell death in feline Niemann-pick type C1 disease. Sci Transl Med. 2015;7(276):276ra226.

46. Vite $\mathrm{CH}, \mathrm{McGowan} \mathrm{JC}$, Niogi $\mathrm{SN}$, et al. Effective gene therapy for an inherited CNS disease in a large animal model. Ann Neurol. 2005;57(3):355-64.

47. Happe H, Peters DJ. Translational research in ADPKD: lessons from animal models. Nat Rev Nephrol. 2014;10(10):587-601.

48. Pei Y, Lan Z, Wang K, et al. A missense mutation in PKD1 attenuates the severity of renal disease. Kidney Int. 2012;81(4):412-7.

49. Wu G, Tian X, Nishimura S, et al. Trans-heterozygous Pkd1 and Pkd2 mutations modify expression of polycystic kidney disease. Hum Mol Genet. 2002;11(16):1845-54

50. Bergmann C, von Bothmer J, Ortiz Bruchle N, et al. Mutations in multiple PKD genes may explain early and severe polycystic kidney disease. J Am Soc Nephrol. 2011;22(11):2047-56

51. Iliuta IA, Kalatharan V, Wang K, et al. Polycystic kidney disease without an apparent family history. J Am Soc Nephrol. 2017;28(9):2768-76.

52. Gansevoort RT, Arici M, Benzing T, et al. Recommendations for the use of tolvaptan in autosomal dominant polycystic kidney disease: a position statement on behalf of the ERA-EDTA working groups on inherited kidney disorders and European renal best practice. Nephrol Dial Transplant. 2016; 31(3):337-48.

53. Soroka S, Alam A, Bevilacqua M, et al. Assessing risk of disease progression and pharmacological Management of Autosomal Dominant Polycystic Kidney Disease: a Canadian expert consensus. Can J Kidney Health Dis. 2017:4:2054358117695784.

\section{Publisher's Note}

Springer Nature remains neutral with regard to jurisdictional claims in published maps and institutional affiliations.

\section{Ready to submit your research? Choose BMC and benefit from:}

- fast, convenient online submission

- thorough peer review by experienced researchers in your field

- rapid publication on acceptance

- support for research data, including large and complex data types

- gold Open Access which fosters wider collaboration and increased citations

- maximum visibility for your research: over $100 \mathrm{M}$ website views per year

At BMC, research is always in progress.

Learn more biomedcentral.com/submissions 